\title{
FOURIER COEFFICIENTS OF CONTINUOUS FUNCTIONS ON COMPACT GROUPS
}

\author{
BARBARA HEIMAN
}

\begin{abstract}
Let $G$ be an infinite compact group with dual object $\Sigma$. Letting $\mathcal{K}_{\sigma}$ be the representation space for $\sigma \in \Sigma, \mathcal{E}^{2}(\Sigma)$ is the set $\left\{A=\left(A^{\circ}\right) \in \Pi \mathscr{B}\left(\mathcal{H}_{0}\right):\|A\|_{2}^{2}=\right.$ $\left.\Sigma_{\sigma} d_{\sigma} \operatorname{Tr}\left(A^{\sigma} A^{\sigma^{*}}\right)<\infty\right\}$. For $A \in \mathcal{E}^{2}(\Sigma)$, we show that there is a function $f$ in $C(G)$ such that $\|f\|_{\infty} \leqslant C\|A\|_{2}$ and $\operatorname{Tr}\left(\hat{f}(\sigma) \hat{f}(\sigma)^{*}\right) \geqslant \operatorname{Tr}\left(A^{\sigma} A^{\sigma^{*}}\right)$ for every $\sigma \in \Sigma$
\end{abstract}

In a 1977 paper [3], K. de Leeuw, Y. Katznelson and J.-P. Kahane proved that every square summable sequence is dominated by the sequence of Fourier coefficients of a continuous function on the circle group, $\mathbf{T}$. As the authors mentioned, this result is true, with the same proof, for any compact abelian group in the role of $\mathbf{T}$ and its dual group in place of the integers, $\mathbf{Z}$. This paper answers the same question for a compact nonabelian group. Using appropriate tools, our proof parallels that of [3]. All notation and terminology used here without explicit definition is as in [2].

Let $G$ be an infinite compact group with dual object $\Sigma$. For each $\sigma \in \Sigma$, let $U^{\sigma}$ be a representation in $\sigma$ and let $\mathcal{H}_{\sigma}$, its representation space, have dimension $d_{\sigma}$. If $\mathscr{B}\left(\mathcal{K}_{\sigma}\right)$ is the space of operators on $\mathcal{K}_{\sigma}$, define \|\|$_{2}$ on $\mathscr{B}\left(\mathcal{K}_{\sigma}\right)$ by $\left\|A^{\sigma}\right\|_{2}=$ $\operatorname{Tr}\left(A^{\sigma} A^{\sigma^{*}}\right)^{1 / 2}$. Let $\mathcal{E}(\Sigma)=\prod_{\sigma \in \Sigma} \Re\left(\mathcal{H}_{\sigma}\right)$ and let $\mathcal{E}^{2}(\Sigma)$ be the set of $A=\left(A^{\sigma}\right) \in \delta(\Sigma)$ satisfying

$$
\|A\|_{2}=\left(\sum_{\sigma} d_{0}\left\|A^{\sigma}\right\|_{2}^{2}\right)^{1 / 2}<\infty .
$$

Finally, $\Gamma$ will designate the compact group $\prod_{\sigma \in \Sigma} \mathcal{Q}\left(d_{\sigma}\right)$, where $\mathscr{Q}\left(d_{\sigma}\right)$ is the group of all unitary operators on $\mathcal{H}_{\sigma}$.

We make use of the following results.

(1) Let $f(V)=\Sigma_{\sigma} d_{\sigma} \operatorname{Tr}\left(B^{\sigma} V^{\sigma}\right)(V \in \Gamma)$ be a finite sum. Then

$$
\int_{\Gamma}|\exp f(V)| d V \leqslant \exp \left(\|B\|_{2}^{2}\right) .
$$

This statement and its proof are similar to [4, Lemma 2].

(2) Suppose $A \in \mathcal{E}^{2}(\Sigma)$. Then, for almost all $V \in \Gamma$,

$$
\sum_{\sigma} d_{\sigma} \operatorname{Tr}\left(A^{\sigma} V^{\sigma} U^{\sigma}(x)\right)
$$

converges for almost every $x \in G[4$, Lemma 8].

Received by the editors May 24, 1982.

1980 Mathematics Subject Classification. Primary 43A30, 43A77, 60B15.

(.)1983 American Mathematical Society $0002-9939 / 82 /(0)(0)-0936 / \$ 02.00)$ 
LEMma 1. Suppose $A \in \varepsilon^{2}(\Sigma)$. For every $V \in \Gamma$, let $f_{V}$ be the function in $L^{2}(G)$ whose Fourier series is

$$
\sum_{\sigma} d_{\sigma} \operatorname{Tr}\left(A^{\sigma} V^{\sigma} U^{\sigma}(x)\right)
$$

Let $\lambda>0$. Then the set

$$
E=\left\{V \in \Gamma: \int_{G} \exp \lambda\left|f_{V}(x)\right| d x \leqslant 2 \exp \left(4 \lambda^{2}\|A\|_{2}^{2}\right)\right\}
$$

has positive $\Gamma$-measure.

Proof. Let $x \in G$ be fixed but arbitrary. Let $S_{n} f_{1}$, be the $n$th partial sum of $f_{1}$ and $S_{n}$, that of $\|A\|_{2}^{2}$. (These partial sums are relative to some arbitrary and fixed enumeration of the set $\left\{\sigma \in \Sigma: A^{\sigma} \neq 0\right\}$.)

Using the inequality $\exp |z| \leqslant \sum_{k=1}^{4} \frac{1}{2}\left|\exp 2 i^{k} z\right|$. the invariance of the Haar integral on $\Gamma$ and (1),

$$
\int_{\Gamma} \exp \lambda\left|S_{n} f_{V}(x)\right| d V \leqslant 2 \exp \left(4 \lambda^{2} S_{n}\right) .
$$

As $x \in G$ was arbitrary,

$$
\int_{G} \int_{\Gamma} \exp \lambda\left|S_{n} f_{V}(x)\right| d V d x \leqslant 2 \exp \left(4 \lambda^{2} S_{n}\right) .
$$

By Fubini's theorem. the order of integration can be reversed. Applying (2) and Fatou's lemma,

$$
\int_{\Gamma} \int_{G} \exp \lambda\left|f_{\mathfrak{V}} \cdot(x)\right| d x d V \leqslant 2 \exp \left(4 \lambda^{2}\|A\|_{2}^{2}\right) .
$$

Consequently, $E$ has positive $\Gamma$-measure.

Lemma 2. Suppose $B \in \mathbb{s}^{2}(\Sigma)$. Let $\varepsilon, \eta>0$ be given and suppose that $\|B\|_{2} \leqslant \varepsilon$. Then there is a choice of $V \in \Gamma$ such that the function $h \in L^{2}(G)$, whose Fourier series is $\Sigma_{\sigma} d_{\sigma} \operatorname{Tr}\left(B^{\sigma} V^{\sigma} U^{o}(x)\right)$, satisfies

$$
\left\|(|h|-\eta)^{+}\right\|_{2} \leqslant 16 \sqrt{2} e^{-1} \varepsilon^{2} \eta^{-1} \exp \left(\frac{-\eta^{2}}{32 \varepsilon^{2}}\right) .
$$

Proof. Let $\lambda=\eta / 8 \varepsilon^{2}$. Invoke Lemma 1 to obtain $V \in \Gamma$ so that

$$
\int_{G} \exp (\lambda|h(x)|) d x \leqslant 2 \exp \left(4 \lambda^{2}\|B\|_{2}^{2}\right) \leqslant 2 \exp \left(\frac{\eta^{2}}{16 \varepsilon^{2}}\right) .
$$

Since

$$
\begin{aligned}
& \sup _{t \geqslant \eta}(t-\eta)^{2} \exp (-\lambda t)=256 e^{-2} \varepsilon^{4} \eta^{-2} \exp \left(\frac{-\eta^{2}}{8 \varepsilon^{2}}\right) \\
&\left\|(|h|-\eta)^{+}\right\|_{2}^{2} \leqslant 256 e^{-2} \varepsilon^{4} \eta^{-2} \exp \left(\frac{-\eta^{2}}{8 \varepsilon^{2}}\right) \int_{G} \exp (\lambda|h(x)|) d x \\
& \leqslant 512 e^{-2} \varepsilon^{4} \eta^{-2} \exp \left(\frac{-\eta^{2}}{16 \varepsilon^{2}}\right) .
\end{aligned}
$$


Lemma 3. Suppose $A \in \mathcal{E}^{2}(\Sigma)$. There exists a function $f \in L^{\infty}(G)$ with $\|f\|_{\infty} \leqslant$ $36\|A\|_{2}$, and $\|\hat{f}(\sigma)\|_{2} \geqslant\left\|A^{\sigma}\right\|_{2}$ for every $\sigma \in \Sigma$.

Proof. Assume that $\|A\|_{2}=1$. Define sequences $\left(\delta_{j}\right)_{j=1}^{\infty},\left(\eta_{j}\right)_{j=1}^{\infty}$, and $\left(\varepsilon_{j}\right)_{j=0}^{\infty}$ by

$$
\delta_{j}=3^{-j} ; \quad \eta_{j}=36 \delta_{j} ; \quad \varepsilon_{0}=1
$$

and

$$
\varepsilon_{j+1}=32 \sqrt{2}\left(1-\delta_{1}\right) e^{-1} \varepsilon_{j}^{2} \eta_{j+1}^{-1} \delta_{j+1}^{-1} \exp \left(\frac{-n_{j+1}^{2}}{32 \varepsilon_{j}^{2}}\right) \quad \text { for } j \geqslant 0
$$

One checks by induction that $\varepsilon_{j} \leqslant 6^{-j}$ for $j \geqslant 0$. Let $s_{0}=0$ and $s_{k}=\sum_{j=1}^{k} \delta_{j}$.

We next define sequences of functions $\left(f_{j}\right)_{j=0}^{\infty},\left(g_{j}\right)_{j=0}^{\infty}$ and $\left(h_{j}\right)_{j=0}^{\infty}$ with $f_{j}=g_{\text {, }}+h$, which satisfy
(a) $\left\|g_{j}\right\|_{\infty} \leqslant 36 s_{j}$;
(b) $\left\|h_{j}\right\|_{2} \leqslant \varepsilon_{j}$;
(c) $\left\|\left(\left|h_{j}\right|-\eta_{j+1}\right)^{+}\right\|_{2} \leqslant \rho_{j}=16 \sqrt{2} e^{-1} \varepsilon_{j}^{2} \eta_{j+1}^{-1} \exp \left(\frac{-\eta_{j+1}^{2}}{32 \varepsilon_{j}^{2}}\right)$;
(d) $\left\|\hat{f}_{j}(\sigma)\right\|_{2} \geqslant\left(1-s_{j}\right)\left\|A^{\sigma}\right\|_{2} \quad$ for $\sigma \in \Sigma$.

Let $g_{0}=0$ and choose $h_{0}$ to satisfy the conclusion of Lemma 2 with $B=A, \varepsilon=\varepsilon_{0}$ and $\eta=\eta_{1}$. Then (a)-(d) are true for $j=0$.

Suppose that $k \geqslant 1$ and that $g_{k-1}$ and $h_{k-1}$ have been selected to satisfy (a)-(d) when $j=k-1$. Define

$$
g_{k}(x)= \begin{cases}36 s_{k} \operatorname{sgn} f_{k-1}(x) & \text { if }\left|f_{k-1}(x)\right|>36 s_{k} \\ f_{k-1}(x) & \text { if }\left|f_{k-1}(x)\right| \leqslant 36 s_{k}\end{cases}
$$

Then (a) holds for $j=k$. Note that if $\left|f_{k-1}(x)\right|>36 s_{k}$, then

$$
\begin{aligned}
\left|f_{k-1}(x)-g_{k}(x)\right| & =\left|f_{k-1}(x)\right|-36 s_{k} \\
& \leqslant\left|g_{k-1}(x)\right|-36 s_{k-1}+\left|h_{k-1}(x)\right|-\eta_{k} \\
& \leqslant\left(\left|h_{k-1}(x)\right|-\eta_{k}\right)^{+} .
\end{aligned}
$$

Thus $\left\|f_{k-1}-g_{k}\right\|_{2} \leqslant \rho_{k-1}$. Let

$$
\Phi=\left\{\sigma \in \Sigma:\left\|\hat{g}_{k}(\sigma)\right\|_{2}<\left(1-s_{k}\right)\left\|A^{\sigma}\right\|_{2}\right\}
$$

For $\sigma \in \Phi$, we have $\left\|\hat{f}_{k-1}(\sigma)-\hat{g}_{k}(\sigma)\right\|_{2} \geqslant \delta_{k}\left\|A^{\sigma}\right\|_{2}$. Define $B \in \mathcal{E}^{2}(\Sigma)$ by

$$
B^{\sigma}= \begin{cases}2\left(1-s_{k}\right) A^{\sigma} & \text { for } \sigma \in \Phi, \\ 0 & \text { otherwise. }\end{cases}
$$


Then.

$$
\begin{aligned}
\|B\|_{2}^{2} & =\sum_{\sigma \in \Phi} d_{\sigma} 4\left(1-s_{k}\right)^{2}\left\|A^{\sigma}\right\|_{2}^{2} \\
& \leqslant 4\left(1-s_{k}\right)^{2} \delta_{k}^{-2}\left\|f_{k-1}-g_{k}\right\|_{2}^{2} \\
& \leqslant 4\left(1-\delta_{1}\right)^{2} \delta_{k}^{-2} \rho_{k-1}^{2}=\varepsilon_{k}^{2} .
\end{aligned}
$$

Thus a function $h_{k}$ can be chosen via Lemma 2 applied to $B, \varepsilon=\varepsilon_{k}$ and $\eta=\eta_{k+1}$ which satisfies both (b) and (c). Finally, if $\sigma \notin \Phi$,

$$
\left\|\hat{f}_{k}(\sigma)\right\|_{2}=\left\|\hat{g}_{k}(\sigma)\right\|_{2} \geqslant\left(1-s_{k}\right)\left\|A^{\sigma}\right\|_{2}
$$

while if $\sigma \in \Phi$,

$$
\left\|\hat{f}_{k}(\sigma)\right\|_{2} \geqslant\left\|B^{\sigma}\right\|_{2}-\left\|\hat{g}_{k}(\sigma)\right\|_{2} \geqslant\left(1-s_{k}\right)\left\|A^{\sigma}\right\|_{2} \text {. }
$$

Therefore (d) holds for $j=k$. This completes the definition of these sequences of functions.

For $j \geqslant 1$,

$$
\left\|f_{j-1}-f_{ر}\right\|_{2} \leqslant\left\|f_{,-1}-g_{j}\right\|_{2}+\|h\|_{2}<2\left(6^{-1}\right) .
$$

so there is a function $f \in L^{2}(G)$ such that $\lim _{, \rightarrow \infty}\left\|2 f_{1}-f\right\|_{2}=0$. Since $\left\|2 g_{1}-f\right\|_{2}$ $\leqslant\left\|2 f_{1}-f\right\|_{2}+2\left\|h_{1}\right\|_{2}$,

$$
\lim _{j \rightarrow \infty}\left\|2 g_{j}-f\right\|_{2}=0 \text {. }
$$

Thus a subsequence of $\left(2 g_{,}\right)_{j=0}^{\infty}$ converges to $f$ pointwise almost everywhere on $G$. Hence

$$
\|f\|_{\infty} \leqslant 2 \varlimsup_{j \rightarrow \infty}\|g,\|_{\infty} \leqslant 36
$$

and, for each $\sigma \in \Sigma$,

$$
\|\hat{f}(\sigma)\|_{2} \geqslant 2 \lim _{j \rightarrow \infty}\left(1-s_{j}\right)\left\|A^{\sigma}\right\|_{2}=\left\|A^{\sigma}\right\|_{2} .
$$

This verifies the lemma.

We are now able to state and prove the main result, which replaces $L^{\infty}(G)$ in Lemma 3 by $C(G)$.

Theorem. Suppose $A \in \mathcal{E}^{2}(\Sigma)$. There exists a function $f \in C(G)$ with $\|f\|_{\infty} \leqslant$ $37\|A\|_{2}$ and $\|\hat{f}(\sigma)\|_{2} \geqslant\left\|A^{\sigma}\right\|_{2}$ for every $\sigma \in \Sigma$.

Proof. Let $\delta=\|A\|_{2} / 36$. Assume $\delta>0$. Let $h \in L^{2}(G)$ have Fourier series

$$
\sum_{\sigma} d_{\sigma} \operatorname{Tr}\left(A^{\sigma} U^{\sigma}(X)\right)
$$

By a factorization theorem, there exist functions $g \in L^{2}(G)$ and $k \in L^{\prime}(G)$ such that

$$
h=k * g \text {; }
$$

$k$ is nonnegative and central in $L^{\prime}(G)$;

$$
\begin{aligned}
& \|k\|_{1}=1 ; \\
& \|h-g\|_{2}<\delta .
\end{aligned}
$$

(See [2, (32.31)], replacing $C(G)$ by $L^{2}(G)$.) 
Invoke Lemma 3 to obtain a function $f_{\infty} \in L^{\infty}(G)$ which satisfies $\left\|f_{\infty}\right\|_{\infty} \leqslant$ $36\|g\|_{2}$ and $\left\|\hat{f}_{\infty}(\sigma)\right\|_{2} \geqslant\|\hat{g}(\sigma)\|_{2}$ for every $\sigma \in \Sigma$. Let $f=k * f_{\infty} \in L^{\prime}(G) * L^{\infty}(G)$ $=C(G)$. Then

$$
\|f\|_{\infty} \leqslant\|k\|_{1}\left\|f_{\infty}\right\|_{\infty} \leqslant 36\|g\|_{2} \leqslant 36\left(\|h\|_{2}+\delta\right)=37\|A\|_{2} .
$$

Since $k$ is central in $L^{1}(G), \hat{k}(\sigma)$ is seen to be a scalar multiple of the identity in ih $\left(\mathcal{K}_{\sigma}\right)$. Write $\hat{k}(\sigma)=c_{\sigma} I_{\sigma}$. Then

$$
\|\hat{f}(\sigma)\|_{2}=\left|c_{\sigma}\right|\left\|\hat{f}_{\infty}(\sigma)\right\|_{2} \geqslant\left|c_{\sigma}\right|\|\hat{g}(\sigma)\|_{2}=\left\|A^{\sigma}\right\|_{2} .
$$

A corollary of our theorem is a generalization of Carleman's theorem for the circle [1]. (See also [2, 37.22(k)] for another proof of this corollary.) For the statement of this corollary, some additional notation is necessary. Let $A \in$ (h) ( $\left.\mathrm{K}_{\mathrm{o}}\right)$ and let $|A|$ be the (unique) positive-definite square root of $A A^{*}$. Let $\left(x_{1}, \ldots x_{d_{0}}\right)$ be the eigenvalues of $|A|$. Define the von Neumann norms on $6\left(t^{\prime}\left(t_{0}\right)\right.$ by

$$
\|A\|_{\phi_{r}}=\left(\sum_{j}\left(x_{j}\right)^{p}\right)^{1 / p} \quad(1 \leqslant p<\infty) .
$$

(Note that \|\|$_{\phi_{2}}$ is the same as \|\|$\left._{2}.\right)$ For $A=\left(A^{o}\right) \in \Sigma(\Sigma)$, define

$$
\|A\|_{p}=\left(\sum_{\sigma} d_{\sigma}\left\|A^{\sigma}\right\|_{\phi_{p}}^{p}\right)^{1 / p} \quad(1 \leqslant p<\infty) .
$$

Let $\stackrel{\Sigma}{p}(\Sigma)=\left\{A \in \varepsilon(\Sigma):\|A\|_{p}<\infty\right\}$.

COROLlARY. There is a continuous function $f$ defined on $G$ for which $\hat{f} \notin \stackrel{s}{(s)}(\Sigma)$ for $1 \leqslant p<2$.

Proof. Let $\left\{\sigma_{2}, \sigma_{3}, \sigma_{4}, \ldots\right\}$ be a countably infinite subset of $\Sigma$, with no repetitions. Let $A^{\sigma_{n}}$ be the $d_{\sigma_{n}} \times d_{\sigma_{n}}$-matrix whose $(1,1)$-entry is $1 / \sqrt{n d_{o_{n}}} \log n$ and whose other entries are zero. Let $A^{\sigma}=0$ for all other $\sigma \in \Sigma$. Then $A \in S^{2}(\Sigma)$. By the preceding theorem, there exists a function $f \in C(G)$ with

$$
\|\hat{f}(\sigma)\|_{2} \geqslant\left\|A^{\sigma}\right\|_{2} \quad \text { for every } \sigma \in \Sigma \text {. }
$$

For every $\sigma \in \Sigma$,

$$
\|\hat{f}(\sigma)\|_{\phi_{r}} \geqslant\|\hat{f}(\sigma)\|_{2} \geqslant\left\|A^{\sigma}\right\|_{2}=\left\|A^{\sigma}\right\|_{\phi_{p}} .
$$

Since

$$
d_{\sigma_{n}}\left\|A^{\sigma_{n}}\right\|_{\phi_{n}}^{p}=d_{\sigma_{n}}\left(\frac{1}{\sqrt{n d_{\sigma_{n}}} \log n}\right)^{p} \geqslant \frac{1}{n \log n}
$$

for all sufficiently large $n, \hat{f} \notin \stackrel{s}{p}(\Sigma)$.

ACKNOWLEDGEMENTS. An earlier version of this paper was written while the author was a graduate student at Kansas State University. I am grateful to the mathematics department there for their support and encouragement. Special thanks are due Karl Stromberg for his guidance. I would also like to thank Edwin Hewitt for his valuable suggestions. 


\section{REFERENCES}

1. T. Carleman, Über die Fourierkoeffizienten einer stetigen Funktion, Acta Math. 41 (1918), 377-384.

2. E. Hewitt and K. A. Ross, Abstract harmonic analysis. II, Grundlehren. Math. Wiss. No. 152. Springer-Verlag, Berlin, Heidelberg and New York, 1970.

3. K. de Leeuw, Y. Katznelson and J.-P. Kahane, Sur les coefficients de Fourier des fonctions continues. C. R. Acad. Sci. Paris Sér. A. 285 (1977), 1001-1003.

4. D. Rider, Random fourier series, Symposia Mathematica No. 22, Academic Press, New York, 1977. pp. $93-106$.

Department of Mathematical Sciences, The College of Wooster, Wooster, Ohio 44691 